\title{
The Art of Human Induced Pluripotent Stem Cells: The Past, the Present and the Future
}

\author{
Xiaogang Zhang ${ }^{1}$, Alejandro De Los Angeles ${ }^{2}$ and Jinqiu Zhang ${ }^{*}, 3$ \\ ${ }^{I}$ Sheng Jing Hospital of China Medical University, Shenyang, 110004, PR China \\ ${ }^{2}$ Harvard Medical School, Boston, MA, 02115, USA \\ ${ }^{3}$ Institute of Medical Biology, 138648, Singapore
}

\begin{abstract}
In 2006, Yamanaka and Takahashi electrified the scientific community by discovering that mouse somatic cells can be converted into embryonic stem cell-like cells by retroviral transduction of four transcription factors: Oct4, Sox2, Klf4, and c-Myc (OSKM). The first generation of mouse induced pluripotent stem (iPS) cells was incompletely reprogrammed, and failed to contribute to germline transmission. Nearly one year later, three groups, including Yamanaka's, improved the reprogramming methodology and generated iPS cells that were in many respects, indistinguishable from ES cells, and also contributed to chimera formation and germline transmission. Shortly thereafter, the successful reprogramming of human somatic cells opened the gate for the development of patient-specific iPS cells for biomedical research and clinical application. Though human iPS cells resemble human ES cells in many aspects, the current iPS cell technologies showed several limitations for clinical usage. First, the efficiency of iPS cell generation is still low and the reprogramming process takes at least two weeks. Second, the virus-delivery of reprogramming factors introduces inconceivable risks of insertional mutagenesis in the genome. Third, given the various strategies for direct reprogramming, it remains difficult to assess the quality of iPS cells generated in each lab and for each patient. These issues should be addressed properly before any iPS cells could be translated into clinic. Here, we review recent progress in human iPS cell technologies, with a focus on the virus-free and integration-free iPS cell generation, which may lead towards the eventual goal of clinical applications.
\end{abstract}

Keyword: Human induced pluripotent stem cells (hiPSCs), reprogramming strategy, hiPSC efficiency.

\section{TOWARDS EFFICIENT PRODUCTION OF HUMAN IPS CELLS}

In late 2007, scientists from the Yamanaka and Thomson laboratories successfully reprogrammed human somatic cells to pluripotency using two sets of transcription factors. Yamanaka and colleagues utilized the same set of transcripttion factors (OSKM) that they had previously demonstrated could reprogram mouse somatic cells to produce iPS cells from human dermal fibroblasts [1]. Thomson and colleagues also reprogrammed human somatic cells, but with the combination of OCT4, SOX2, NANOG and LIN28 (OSNL) [2]. Both groups have shown that human iPS cells resemble human ES cells in many aspects including morphology, proliferation, pluripotency markers, gene expression profiles, epigenetic status, and ability to differentiate into three germ layers. The achievement of human iPS cells holds great promise for regenerative medicine. It may have potential to replace human ES cells in cell therapy, which is hindered by immuno rejection and ethical issues. However, the efficiency of traditional iPS cell generation is only $0.001-0.01 \%$ of starting cells and the requirement for virus transduction prohibits its application in clinical therapy.

The slow and inefficient process of deriving human iPS cells motivated efforts to improve reprogramming. Addition

*Address correspondence to this author at the Institute of Medical Biology, 138648, Singapore; Tel: (65) 6407 0218; Fax: (65) 6464 2048; E-mail: jinqiu.zhang@imb.a-star.edu.sg of Large $\mathrm{T}$ and TERT to either set of the reprogramming factors increased the efficiency of iPS cell generation by 2370 fold [3]. In another study, p53 knock down by siRNA delivery and introduction of UTF1 to OSKM cocktail enhanced the reprogramming efficiency to approximately 100 fold [4]. Recently a series of papers have extended the observation and show that ARF-p53 pathways are ratelimiting barriers in the reprogramming process [5-9]. The expression of "Yamanaka factors" induces expression of p53, p16 and p21, which results in DNA-damage and senescence. Releasing the barrier by transcriptional depletion of p21 or p53 increases iPS generation by approximately 100-fold. However, the convergent roles of c-Myc, Large T, TERT and lack of p53 promote immortalization, which may lead to DNA damage, genomic instability and tumorigenesis of iPS cells. In addition, with these potential oncogenes as the reprogramming factors, there is a risk of re-activation during iPS cell differentiation. Indeed, re-activation of c-myc was reported to promote tumor formation in $20 \%$ of iPS cell chimeric mice [10]. Thus replacement of these factors is necessary for clinical applications.

\section{ONCOGENE REPLACEMENT AND REPROGRAMM- ING EFFICIENCY}

The replacement of KLF4 and c-Myc with NANOG and LIN28 indicated that successful targeting of OCT4 and SOX2 to appropriate binding sites is sufficient for 
reactivation of the pluripotent transcriptional network, and that NANOG- and LIN28-mediated events can replace KLF4- and c-Myc in direct reprogramming [2]. Later, several groups demonstrated that c-Myc is dispensable in this process $[11,12]$. Chimeras made from myc-free iPS cells have reduced tumorigenicity. However, without c-Myc, reprogramming efficiency is 100 fold lower and the time for the appearance of stem cell colonies is longer. To overcome this problem, Huangfu et al. screened several histone deacetylase and DNA methyltransferase inhibitors, and identified valproic acid (VPA) as most effective in replacing c-Myc for iPS cell production [13]. The use of three factors OSK in the presence of VPA increased the reprogramming efficiency of human primary fibroblasts to $1 \%$, a 1000 fold increase compared to previous reports. This method is repeatable and in our experience a relatively efficient way of iPS cell generation by virus transduction. The detailed modifications of protocol are summarized in Table 1.

Using modified protocol Huangfu et al. were able to get iPS cells from transduction of two factors, OCT4 and SOX2, and efficiency equivalent to OSK with other methods. The efficacy of the protocol indicates that the starting epigenetic status and particular histone acetylation may be ratedetermining for direct reprogramming.

\section{CELL TYPES AND REPROGRAMMING EFFI- CIENCY}

The type of somatic cell used for deriving iPS cells may influence the quality and efficiency of reprogramming. In addition to fibroblasts, mouse iPS cells have been generated from hepatocytes, gastric epithelial cells [14], pancreatic cells [15], neural stem cells [16,17] and B lymphocytes [18]. Human iPS cells have been generated from fibroblasts [1,2], keratinocytes [19], mesenchymal cells [41] and blood progenitor cells [20]. Aasen et al. compared reprogramming of human keratinocytes and foreskin fibroblasts obtained from the same person. Using the same batch of retrovirus OSKM, the infection of keratinocytes yielded iPS cells at an efficiency of $1 \%, 100$ fold higher than in fibroblast $(0.01 \%)$. Moreover, iPS cells emerged 10 days after infection, as compared to 21-30 days for fibroblast. Cells showed similar characteristics to ES cells such as tight cell-cell contact, surface expression of E-cadherin and higher levels of endogenous KLF4 and c-Myc. It has been suggested that because keratinocytes and ES/iPS cells are epithelial-like cells with a similar epigenetic state, keratinocytes do not have to undergo a mesenchymal-epithelial transition necessary for fibroblasts [19]. Interestingly, small amounts of keratinocytes obtained from the follicle cells of a single human hair were sufficient to generate iPS cells. In clinical settings, hair follicle cells and blood are more convenient source of cells than fibroblasts, which need skin biopsy.

\section{REDUCED GENOME INTEGRATION SITES WITH SINGLE CASSETTE VECTORS}

Direct reprogramming of fibroblasts via lentiviral or retroviral transduction requires high virus titer with 15-20 proviral integration and $30 \%-90 \%$ transduction efficiency [21]. The comparatively low reprogramming efficiency may be partially due to transduction with separate vectors allowing integration of different numbers of proviruses for each factor. The cells that do not carry all four factors fail to form iPS cells [22,23]. Thus efficiency is lower than expected. In another study using dox-inducible lentivirus reprogramming, while primary iPS cells were differentiated into secondary fibroblasts in the absence of DOX, the secondary fibroblasts were reprogrammed into iPS cells with 100 fold higher efficiency when cultured in the presence of DOX [24]. It indicates that the correct stoichiometry of the four reprogramming factors may be critical for high efficiency and reprogramming using a single cassette of four factors displays is advantageous. In addition, reducing the number of viral integration sites enhances the chance for subsequent removal of the exogenous genes.

Single cassette vectors have been described that are polycistronic lentiviral vectors expressing multiple genes simultaneously via self-cleaving $2 \mathrm{~A}$ peptide. The defined factors OSKM were cloned in frame with 2A peptide separating each factor, which support near equimolar protein expression. Stem cells generated by this method were found

Table 1. Comparison of Two Method of Retrovirus-Mediated iPS Generation

\begin{tabular}{|c|c|c|}
\hline & Takahashi et al. & Huangfu et al. \\
\hline Plasmids used & pMXs-O,S,K,M & pMXs-O,S,K,M \\
\hline Packaging system & PLAT-E cells & Gag-pol, VSV-G plus $293 \mathrm{~T}$ cells \\
\hline Virus used for infection & $\begin{array}{l}\text { Ectropic retrovirus produced in PLAT-E cells, concentration of } \\
\text { virus is not recommended }\end{array}$ & Concentrated VSV-G pseudotyped retrovirus \\
\hline $\begin{array}{l}\text { Cells used for } \\
\text { reprogramming }\end{array}$ & $\begin{array}{l}\text { Human fibroblast cells pre-infected with Lentivirus expressing } \\
\text { mouse receptor of retrovirus, Slc } 7 \mathrm{a} 1 \text {, before transduction with } 4 \\
\text { factors }\end{array}$ & Human fibroblast cells without modifications \\
\hline $\begin{array}{l}\text { Culture conditions after } \\
\text { viral transduction }\end{array}$ & $\begin{array}{l}\text { 1). Cells keep in fibroblast medium till } 7 \text { days and switch to } \\
\text { human ES medium. } \\
\text { 2). Replate human fibroblast cells on MEF feeders at day } 6 \text { after } \\
\text { infection. }\end{array}$ & $\begin{array}{l}\text { 1). Switch to human ES medium immediately after } \\
\text { infection. } \\
\text { 2). Valproic acid ( } 0.5-1 \mathrm{mM}) \text { was added for } 1-2 \text { weeks. } \\
\text { 3). The infected cells were allowed to grow undisturbed } \\
\text { without splitting and replating on feeder cells. }\end{array}$ \\
\hline $\begin{array}{l}\text { Reprogramming } \\
\text { efficiency }\end{array}$ & $0.02 \%$ & $1 \%$ \\
\hline
\end{tabular}

Abbreviations: O, Oct3/4; S, Sox2; K, Klf4; M, c-Myc. 
to have a reduced number of integration sites (1-2 sites in all of the colonies tested) $[25,26]$. Subsequently, the reprogramming factors can be removed by $\mathrm{Cre}$ mediated excision with some part of the vector backbone still in the genome [27]. However, given the fact that these lentivirus vectors are usually large in size (12-13kbp), virus packaged from these vectors has low transduction efficiency, compromising the advantage of a single cassette. Furthermore, the reported reprogramming efficiency for iPS cell generation is $0.5 \%$ $1 \%$, which did not show a great advantage over other methods.

\section{TOWARDS DNA-FREE iPS CELLS}

\section{Virus-Free iPS Cells}

Lentiviral and retroviral based reprogramming result in multiple transgenes, which may lead to an increased risk of mutagenesis. For example, previous studies have shown that retroviruses integration can activate endogenous genes that cause cancer. iPS cells generated through these methods would not be acceptable for clinical use.

Several groups have demonstrated that genome integration is not necessary for iPS cell production. Aoi and colleagues found that iPS clones do not share common insertional provirus sites, indicating that site-specific insertional mutagenesis is not necessary for the reprogramming process [14]. Another elegant study utilizing doxycyclineinducible lentiviral constructs for delivery of OSKM genes demonstrated that reprogramming factors were only required for a period of two weeks for iPS cell generation, after which the overexpressed genes were silenced [24]. These studies helped pave the way for developing technologies to generate iPS cells without viral integration, with an understanding of how long and at what levels the reprogramming factors needed to be expressed.

With these findings in mind, early attempts to generate iPS cells without viral integration include the repeated transient transfection of plasmid-based vectors into mouse embryonic fibroblasts [28], and the use of adenoviruses in mouse liver cells [29] (summarized in Table 2). However, in both cases, the reprogramming efficiency was extremely low with slower kinetics, and no iPS cells have been generated from human cells using such methods.
Another virus-free delivery method is the piggyBac transposition system, which serves as a vehicle for up to $10 \mathrm{~kb}$ cargo capacity without losing transposition efficiency. With this tool, Nagy and colleagues generated both mouse and human iPS cells by transfecting fibroblasts with the piggyBac transposase and a polycistronic plasmid encoding the four "Yamanaka factors" linked by 2A sequences [30]. Their approach combined the advantages of viral integration with the need to have integration-free cells. By allowing the reprogramming factors to integrate into the genome, cells could maintain the appropriate levels of reprogramming factors through several cell divisions, allowing the gradual process of reprogramming to occur at a meaningful efficiency. By using a system that allows seamless excision of the factors, they could generate mouse iPS cells without persistent transgene expression and reduce the risk of insertional mutagenesis. However, the strategy of removing integrated transgenes requires an additional step that might prevent widespread clinical application of iPS cells. Given the slow growth rate of human iPS cell, complete removal of the transgenes has not been reported yet.

\section{Virus-Free and Integration-Free iPS Cells}

To simplify the derivation of integration-free human iPS cells, Yu et al. transfected somatic cells with an oriP/EBNA1 (Epstein-Barr nuclear antigen-1)-based episomal vector [31]. These plasmids can be transfected without the need for viral packaging, and replicate without integration in to the genome. The stable extrachromosomal expression of transgenes can be maintained by drug selection during reprogramming and they can be subsequently removed from cells by culturing in the absence of drug selection. When SV40 large T (SV40LT) was included in their cocktail, along with Oct4, Sox2, Klf4, c-Myc, Nanog, and Lin28, the authors were able to generate iPS cells from human foreskin fibroblasts in two independent experiments. While no integration was observed, clones were fully reprogrammed and had normal karyotypes. However, this virus-free and integration-free method required the use of SV40 Large T, and was still hindered by a low reprogramming efficiency. As the stable transfection efficiency for primary cells is 100 fold lower than virus infection, the efficiency for iPS cell generation by episomal vector is less than $0.001 \%$. In addition, the continued use of nucleic acid delivery and its

Table 2. Integration-Free Method for iPS Cell Generation

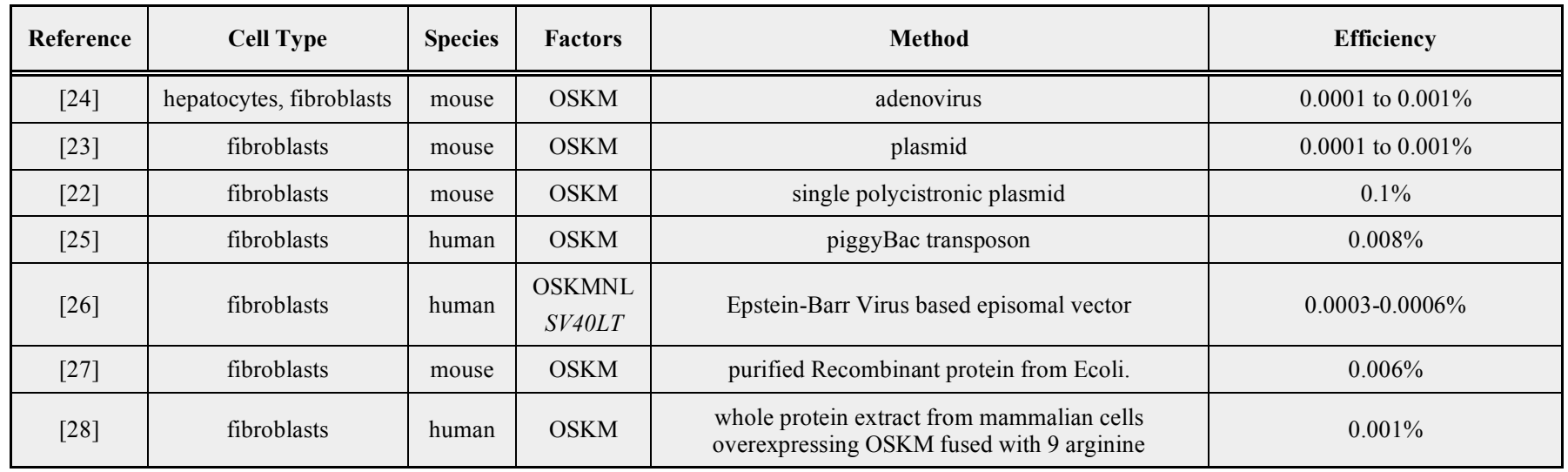

Abbreviations: O, Oct3/4; S, Sox2; K, Klf4; M, c-Myc; N, Nanog; L, Lin28; SV40LT, SV40 large T gene. 
associated risk of genomic integration means that a simpler method with improved efficiency is still needed before integration-free iPS cells can be more widely used.

\section{DNA-Free iPS Cells}

Until recently, all methods to generate iPS cells required the use of genetic materials. To avoid introducing exogenous genetic modifications into target cells, both Sheng Ding and Kim's lab used the original four reprogramming proteins fused with a cell penetrating peptide to generate DNA-free iPS (piPS) cells $[32,33]$. The two protocols differ in several important respects. Sheng Ding's method required the use of VPA and proteins were expressed in E. coli inclusion body, which were then solubilized, refolded and purified. They worked on mouse fibroblasts and obtained mouse piPS cells that fulfilled all the criteria for pluripotent stem cells including chimera formation and germline transmission. However, attempts to use only protein cocktails without VPA, which may exert potential off-target effects, failed to generate any mouse piPS cells. Furthermore, data on human piPS cells is not available. Kim's method used whole protein extracts of HEK293 cells that expressed high levels of the four "Yamanaka factors" and generated human iPS cells without the use of any small molecules such as VPA. In both protocols, repeated transduction of proteins to somatic cells led to the successful generation of iPS cells free of nucleic acid delivery. Though with low efficiency (0.001-0.006\%) and long period of process (5-8 weeks), these methods represent great advances over previous protocols for future potential clinical application by eliminating the risk of genome alteration by exogenous genetic sequences (Fig. 1). Moreover, the E. coli and mammalian expression of reprogramming factors facilitates the large-scale production of recombinant protein and make quality control of the iPS cell generation possible in future.

\section{FUTURE DIRECTIONS}

The generation of human DNA-free iPS cells by Kim's team is an important milestone toward the ultimate goal of customized cell therapy. Future clinical reprogramming can be improved technically in the following aspect. First, to improve reprogramming efficiency, the four reprogramming proteins may be purified from the mammalian expressing cells instead of the current used whole-protein extracts of HEK293 cells, which reduced the efficacy of reprogramming factors by HEK293 cell components. Furthermore, previous experiments have shown that mouse factors can also be used to generate human iPS cells from somatic cells with similar efficiency $[13,26]$. This suggests that the entire protein sequences of the four factors are not required, and that the homologous domains controlling the activation of the target genes, may be sufficient for reprogramming. Thirdly, small molecules have been shown to replace some of the factors, such as Valproic acid (VPA) for klf4 and c-Myc [13], histone methyltrasferase inhibitor BIX-01294 and TGF-beta
A

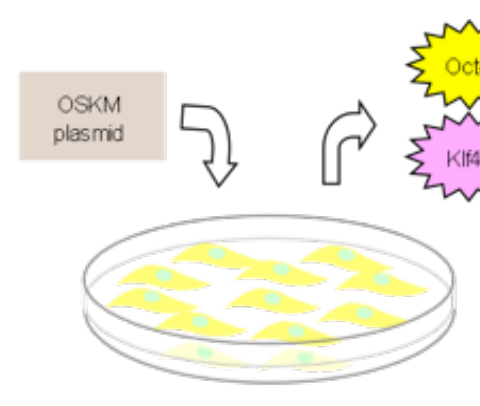

HEK293T cells
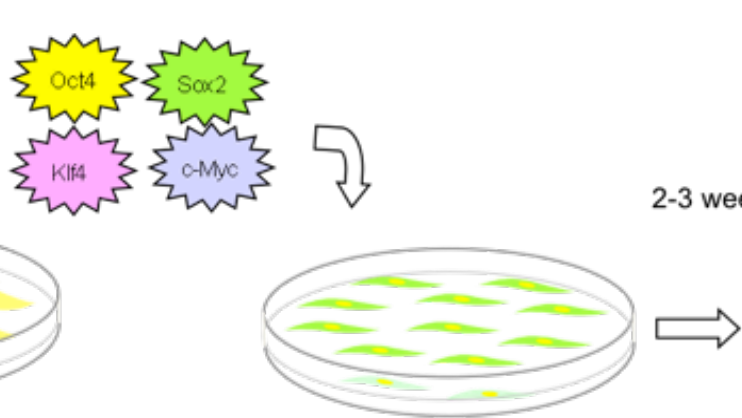

Human fibroblasts
2-3 weeks

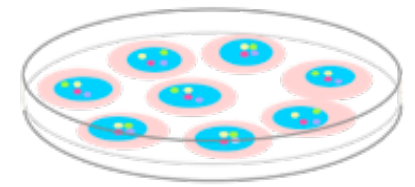

iPS cells with viral integration

8 weeks

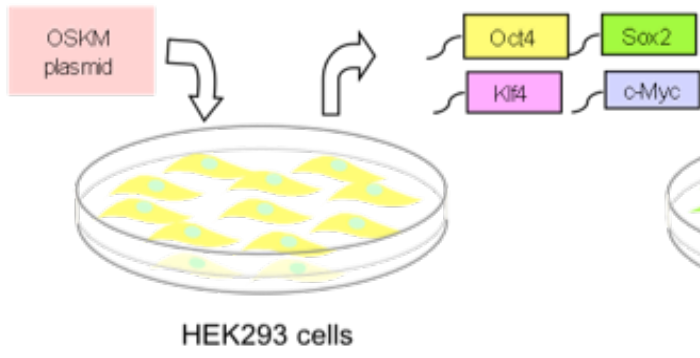

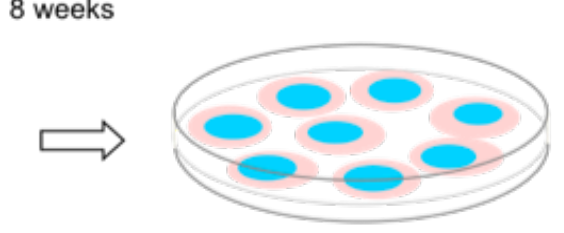

iPS cells with NO integration

Fig. (1). Comparison of retrovirus and protein mediated reprogramming. A. Retrovirus plasmid pMXs-Oct4, Sox2, Klf4, c-Myc was transfected together with packaging plasmid gag-pol and VSV-G in HEK293T cells to produce retrovirus. Retrovirus carrying Oct4, Sox2, Klf4 and c-Myc were transduced in human fibroblast cells. iPS cells were derived in 2-3 weeks with genomic viral integrations. B. Mammalian overexpression vector pcDNA3.1 coding for Oct4, Sox2, Klf4, c-Myc cDNA are transfected in HEK293 cells to generate stable cell lines overexpressing Oct4,Sox2, Klf4 and c-Myc proteins fused with a cell membrane penetrating tag, 9 arginine. The whole protein extract of each stable HEK293 cell lines of the 4 factors were incubated with human fibroblast cells. After 8 weeks, the iPS cells were generated with no genomic integration. 
inhibitor for Sox2 [34,35]. However, reprogramming with exclusive small molecules is still under development.

In addition to cell therapy, iPS cells will be useful for understanding disease biology and drug development. iPS cells have been generated from patients with amyotrophic lateral sclerosis (ALS) [36], spinal muscular atrophy (SMA) [37], Parkinson's disease (PD) [38], $\beta$-thalassemia [39] and Rett syndrome [40]. In addition, lines have been produced from donors with adenosine deaminase deficiency-related severe conbined immunodeficiency (ADA-SCID), Shwachman-Bodian-Diamond syndrome (SBDS), Gaucher disease (GD), Duchenne (DMD), Becker muscular dystrophy (BMD), Huntington disease (HD), Diabetes Mllitus type 1, Down syndrome (DS) and a carrier of Lesch-Nyhan syndrome [41]. Some iPS cell lines have been differentiated to cell lineages that recapitulate the defect of patient cells. This could serve as a patient-specific disease model for drug screening, which is otherwise experimentally not accessible.

The longer-term use of iPS cells for cell therapeutics is partly driven by the possibility that genetic mutations can be corrected by homologous recombination towards curing some genetic diseases by cell transplantation. The recent work by Raya et al. has characterized genetically corrected iPS cells from patients with Fanconi anaemia (FA) [42]. By Lentivirus expression of FANCA gene into the fibroblasts of FA patients, the genetically corrected fibroblasts were reprogrammed into iPS cells that gave rise to hematopoietic progenitors. These hematopoietic progenitors of myeloid and erythroid lineages were phenotypically normal/disease-free and could potentially be used to rescue bone marrow failure syndrome of FA patients. Though the genetic correction was done by Lentivirus expression rather than homologous recombination and failed in animal engraftment due to possible technical limitations, this effort has brought the realization of cell therapy for genetic diseases closer.

\section{CONCLUDING REMARKS}

The first iPS cells were derived from murine somatic cells four years ago, and from human cells a year later. Despite great advances, much still needs to be clarified before iPS cells can be fully utilized in basic research and clinical therapy. The reprogramming of somatic cells by forced expression of defined factors appears to be a random process that requires many progressive nonspecific epigenetic remodeling events over a prolonged period of time; usually 2 weeks for mouse cells and $3-4$ weeks for human cells. What exactly happens during this period of time remains unknown. The much faster reprogramming by somatic cell nuclear transfer (SCNT) requires only 24 hours for OCT4 activation [43], likely involves different key reprogramming mechanisms. Understanding the key events that lead to the rare but robust reprogramming of somatic cells will accelerate efforts to translate iPS cells into the clinic.

\section{ACKNOWLEDGEMENTS}

We thank Dr. Jeremy Crook and Dr. Oliver Dreesen for critically reviewing the manuscript.

\section{REFERENCES}

[1] Takahashi K, Tanabe K, Ohnuki M, et al. Induction of pluripotent stem cells from adult human fibroblasts by defined factors. Cell 2007; 131: 861-72.

[2] Yu J, Vodyanik MA, Smuga-Otto K, et al. Induced pluripotet stem cell lines derived from human somatic cells. Science 2007; 318: 1971-20.

[3] Mail P, Ye Z, Hommond HH, et al. Improved efficiency and pace of generating induced pluripotent stem cells from human adult and fetal fibroblasts. Stem Cells 2008; 26: 1998-2005.

[4] Zhao Y, Yin X, Qin H, et al. Two supporting factors greatly improve the efficiency of human iPS cell generation. Cell Stem Cell 2008; 3: 475-9.

[5] Hong H, Takahashi K, Ichisaka T, et al. Suppression of induced pluripotent stem cell generation by the p53-p21 pathway. Nature 2009; 460: 1132-5.

[6] Marion RM, Strati K, Li H, et al. A p53-mediated DNA damage response limits reprogramming to ensure iPS cell genomic integrity. Nature 2009; 460: 1149-53.

[7] Utikal J, Polo JM, Stadtfeld M, et al. Immortalization eliminates a roadblock during cellular reprogramming into iPS cells. Nature 2009; 460: 1145-8.

[8] Li H, Collado M, Villasante A, et al. The Ink4/ARF locus is a barrier for iPS cell reprogramming. Nature 2009; 460: 1136-9.

[9] Kawamura T, Suzuki J, Wang YV, et al. Linking the p53 tumour suppressor pathway to somatic cell reprogramming. Nature 2009; 460: 1140-4

[10] Okita K, Ichisaka T, Yamanaka S. Generation of germline competent induced pluripotent stem cells. Nature 2007; 448: 313-7.

[11] Nakagawa M, Koyanagi M, Tanabe K, et al. Generation of induced pluripotent stem cells without Myc from mouse and human fibroblast. Nat Biotechnol 2008; 26: 101-6.

[12] Wernig M, Meissner A, Cassady JP, et al. c-Myc is dispensable for direct reprogramming of mouse fibroblasts. Cell Stem Cell 2008; 2: 10-12.

[13] Huangfu K, Maehr R, Guo W, et al. Induction of pluripotent stem cells by defined factors is greatly improved by small-molecule compounds. Nat Biotechnol 2008; 26: 795-7.

[14] Aoi T, Yae K, Nakagawa M, et al. Generation of pluripotent stem cells from adult mouse liver and stomach cells. Science 2008; 321 : 699-702.

[15] Stadtfeld M, Brennand K, Hochedlinger K. Reprogramming of pancreatic beta cells into induced pluripotent stem cells. Curr Biol 2008; 18: 890-4.

[16] Eminli S, Utikal J, Arnold K, Jaenisch R, Hochedlinger K. Reprogramming of neural progenitor cells into induced pluripotent stem cells in the absence of exogenous Sox 2 expression. Stem Cells 2008; 26: 2467-74.

[17] Kim JB, Zaehres H, Wu G, et al. Pluripotent stem cells induced from adult neural stem cells by reprogramming with two factors. Nature 2008; 454: 646-50.

[18] Hanna J, Markoulaki S, Schorderet P, et al. Direct reprogramming of terminally differentiated mature B lymphocytes to pluripotency. Cell 2008; 133: 250-64.

[19] Aasen T, Raya A, Barrero MJ, et al. Efficient and rapid generation of induced pluripotent stem cells from human keratinocytes. Nature Biotechnol 2008; 26: 1276-84

[20] Loh YH, Agarwal S, Par IH, et al. Generation of induced pluripotent stem cells from human blood. Blood 2009; 113: 5476-9.

[21] Takahashi K, Yamanaka S. Induction of pluripotent stem cells from mouse embryonic and adult fibroblast cultures by defined factors. Cell 2006; 126: 663-76.

[22] Lowry WE, Richter L, Yachechko R, et al. Generation of human induced pluripotent stem cells from dermal fibroblasts. Proc Natl Acad Sci USA 2008; 105: 2883-8.

[23] Papapetrou EP, Tomishima MJ, Chambers SM, et al. Stoichiometric and temporal requirements of Oct4, Sox2, Klf4, and c-Myc expression for efficient human iPS cell induction and differentiation. Proc Natl Acad Sci USA 2009. [Epub ahead of print].

[24] Maherali N, Ahfeldt T, Rigamonti A, Utikal J, Cowan C, Hochedlinger K. High-efficiency system for the generation and study of human induced pluripotent stem cells. Cell Stem Cell 2008; 3: 340-5 
[25] Sommer C, Stadtfeld M, Murphy G, Hochedlinger K, Kotton DN, Mostoslavsky G. Induced pluripotent stem cell generation using a single lentiviral stem cell cassette. Stem Cell 2009; 27: 543-9.

[26] Carey BW, Markoulaki S, Hanna J, Saha K, Gao Q, Mitalipova M. Reprogramming of murine and human somatic cells using a single polycistronic vector. Proc Natl Acad Sci USA 2009; 106: 157-62.

[27] Kaji K, Norrby K, Paca A, Mileikovsky M, Mohseni P, Woltjen K. Virus-free induction of pluripotency and subsequent excision of reprogramming factors. Nature 2009; 458: 771-5.

[28] Okita K, Nakagawa M, Hyenjong H, Ichisaka T, Yamanaka S. Generation of mouse induced pluripotent stem cells without viral vectors. Science 2008; 322: 949-53.

[29] Stadtfeld M, Nagaya M, Utikal J, Weir G, Hochedlinger K. Induced pluripotent stem cells generated without viral integration. Science 2008; 322: 945-9.

[30] Woltjen K, Michael IP, Mohseni P, et al. piggyBac transposition reprograms fibroblasts to induced pluripotent stem cells. Nature 2009; 458: 766-70.

[31] Yu J, Hu K, Smuga-Otto K, et al. Human induced pluripotent stem cells free of vector and transgene sequences. Science 2009; 324: 797-801.

[32] Zhou H, Wu S, Joo JY, et al. Generation of induced pluripotent stem cells using recombinant proteins. Cell Stem Cell 2009; 4: 3814.

[33] Kim D, Kim CH, Moon JI, et al. Generation of human induced pluripotent stem cells by direct delivery of reprogramming proteins. Cell Stem Cell 2009; 4: 472-6.
[34] Shi Y, Do JT, Desponts C, Hahm HS, Scholer HR, Ding S. A combined chemical and genetic approach for the generation of induced pluripotent stem cells. Cell Stem Cell 2008; 2: 525-8.

[35] Ichida JK, Blanchard J, Lam K, et al. A small-molecule inhibitor of Tgf-beta signaling replaces Sox2 in reprogramming. Cell Stem Cell 2009; 5: 491-503.

[36] Dimos JT, Rodolfa KT, Niakan KK, et al. Induced pluripotent stem cells generated from patients with ALS can be differentiated into motor neurons. Science 2008; 321: 1218-21.

[37] Ebert AD, Yu J, Rose FF Jr, et al. Induced pluripotent stem cells from a spinal muscular atrophy patient. Nature $2009 ; 457: 277-80$.

[38] Soldner F, Hockemeyer D, Beard C, et al. Parkinson's disease patient-derived induced pluripotent stem cells free of viral reprogramming factors. Cell 2009; 136: 964-77.

[39] Ye L, Chang JC, Lin C, Sun X, Yu J, Kan YW. Induced pluripotent stem cells offer new approach to therapy in thalassemia and sickle cell anemia and option in prenatal diagnosis in genetic diseases. Proc Natl Acad Sci USA 2009; 106: 9826-30.

[40] Hotta A, Cheung AY, Farra N, et al. Isolation of human iPS cells using EOS lentiviral vectors to select for pluripotency. Nat Methods 2009; 6: 370-6.

[41] Park IH, Arora N, Huo $\mathrm{H}$, et al. Disease-specific induced pluripotent stem cells. Cell 2008; 134: 877-86.

[42] Raya A, Rodríguez-Pizà I, Guenechea G, et al. Disease-corrected haematopoietic progenitors from Fanconi anaemia induced pluripotent stem cells. Nature 2009 [Epub ahead of print].

[43] Boiani M, Eckardt S, Scholer HR, McLaughlin KJ. Oct4 distribution and level in mouse clones: consequences for pluripotency. Genes Dev 2002; 16: 1209-19.

(C) Zhang et al.; Licensee Bentham Open.

This is an open access article licensed under the terms of the Creative Commons Attribution Non-Commercial License (http://creativecommons.org/licenses/by$\mathrm{nc} / 3.0 /$ ), which permits unrestricted, non-commercial use, distribution and reproduction in any medium, provided the work is properly cited. 